\title{
РАЗВИТИЕ МАЛОГО И СРЕДНЕГО ПРЕДПРИНИМАТЕЛЬСТВА В КОНТЕКСТЕ СЕРВИСИЗАЦИИ И ИНТЕЛЛЕКТУАЛИЗАЦИИ ЭКОНОМИКИ БЕЛАРУСИ
}

\author{
Ю.А. Медведева, М.П. Томкович*
}

На основе обзора литературных источников раскрыты вопросы влияния инноваций на малое и среднее предпринимательство (МСП) в современных условиях, в том числе с учетом процессов сервисизации и цифровизации. Проведен анализ развития МСП в Беларуси. Рассмотрен ряд государственных программных документов с позиции развития малого и среднего предпринимательства в данном контексте. Установлено, что они достаточно фрагментарны и ориентированы преимущественно на количественные параметры развития этой сферы экономики. Качественная сторона развития субъектов МСП фактически остается вне фокуса государственного управления, что не позволяет говорить о выраженном векторе государственной политики. Уделено внимание вопросам функционирования системы государственной поддержки МСП. Проанализирован инструментарий развития МСП в Европейском союзе, включая реализацию Новой промышленной стратегии и концепции «двойного перехода», предусматривающих достижение климатической нейтральности и цифровое лидерство.

Ключевые слова: МСП, инновации, сервисизация, интеллектуализация, зарубежный опыт.

JEL-классификация: L26, O14, O30, O38.

DOI: $10.46782 / 1818-4510-2021-4-138-148$

Материал поступил 3.12.2021 2.

Современный этап развития экономики и общества характеризуется усилением роли и значимости малого и среднего предпринимательства, которое становится неотъемлемым звеном в создаваемых цепочках добавленной стоимости, что способствует повышению эффективности производства и, как следствие, ускоренному социально-экономическому развитию.

Динамичное развитие малого предпринимательства является необходимым условием стабильного функционирования экономики любого государства. Необходимо отметить, что в развитых странах мира именно малый бизнес во многом определяет темпы экономического роста и структуру валового внутреннего продукта.

Благодаря своей быстрой адаптивности, по сравнению с крупными корпорация- ми и предприятиями, МСП способны расширять сферу приложения труда, создавать новые возможности не только для трудоустройства, но прежде всего для расширения поля самостоятельной деятельности населения, развертывания его творческих сил и использования свободных производственных мощностей.

Значимость малого и среднего бизнеса еще более будет возрастать в контексте прогрессирующей сервисизации экономики. Если раньше услугам отводилась преимущественно вспомогательная роль сопровождения материальных благ, то сегодня именно сервисная составляющая в значительной степени определяет конкурентоспособность как отдельного товара, так и всей экономики (Томкович, 2021). В этой ситуации кардинально растет ценность индивидуального подхода к

* Медведева Юлия Александровна (у_medvedeva@mail.ru), кандидат экономических наук, Белорусский институт системного анализа и информационного обеспечения научно-технической сферы (г. Минск, Беларусь). https://orcid.org/00000001-8089-8356;

Томкович Михаил Петрович (tomkovich.michael@gmail.com), кандидат экономических наук, Научно-исследовательский экономический институт Министерства экономики Республики Беларусь (г. Минск, Беларусь). https://orcid.org/ 0000-0001-5957-0321 
удовлетворению разнообразных потребностей экономических субъектов, который традиционно являлся сильной стороной МСП. Все это в совокупности обуславливает несомненную важность вопросов повышения значимости небольшого бизнеса в экономике Республики Беларусь с акцентом на развитие интеллектуальной и сервисной составляющих.

\section{Взгляд на инноващии в МСП: краткий обзор зарубежной литературы}

Важность инновационной составляющей в развитии МСП признается в подавляющем большинстве исследований, посвященных данной проблематике, как в Беларуси, так и за рубежом. Особенно этот вопрос актуализировался в контексте цифровизации и сервисизации значительной части бизнес-процессов. Например, практически каноничными стали изыскания Шумпетера (1982), который рассматривал инновационность, постоянное стремление к новаторству и творческий подход в качестве важнейших черт предпринимателя.

На современном этапе при объяснении значимости инноваций для развития предпринимательства достаточно популярна концепция «голубого океана», которую предложили Ким и Мауборгнэ (Kim, Mauborgne, 2005).

Примеры можно продолжать, однако, вне зависимости от названий теоретических конструктов и некоторых различий в их содержании, они все имеют как минимум одну общую составляющую, а именно - неразрывность предпринимательства как явления и стремления к нововведениям (новациям). Аксиоматичным стало утверждение, что фундаментом успешного предпринимательства является поиск новой идеи относительно товара (услуги), используемой технологии, бизнес-модели или иных составляющих, делающих предложение на рынке конкурентным.

Несмотря на богатую историю изучения предпринимательства, вопросам его развития и анализа факторов, определяющих его эффективность, по сей день уделяется достаточно много внимания исследователей по всему миру.

Говоря о новациях, следует упомянуть работу (Rosenbusch, Brinckmann, Bausch, 2011), опирающуюся на эмпирические исследования 21270 фирм и наглядно доказывающую положительное влияние инноваций на субъекты МСП и, в частности, их производительность. При этом речь следует вести как о важности внедрения инноваций в предлагаемые товары и услуги, так и необходимости общей ориентации на инновации при выстраивании бизнес-модели. В частности, в работе (Latifi, Nikou, Bouwman, 2021) показано, что инновационные бизнес-модели (ИБМ) в субъектах МСП напрямую влияют на рост их эффективности, организационные возможности и увеличение доходов. Акцентируется внимание также на том, что внедрение ИБМ несет в себе потенциальные риски, связанные с фундаментальными и необратимыми изменениями в ключевых компонентах бизнес-модели компании, что требует от ее менеджмента взвешенного подхода и глубокой проработки всех составляющих процесса внедрения инноваций.

В условиях очевидной необходимости внедрения инновационной составляющей в деятельность МСП существует проблема недостаточности ресурсов у бизнеса, включая производственные мощности, маркетинговые возможности, каналы сбыта и международные контакты. Решению этой проблемы посвящена работа (Sağ, Sezen, Güzel, 2016) в которой основной акцент сделан на идее отрытых инноваций, предполагающей сотрудничество субъектов МСП с другими организациями для получения внешних знаний, ресурсов и дополнительных активов, которые им необходимы для разработки и коммерциализации своих изобретений.

Значительный пласт исследований посвящен вопросам адаптации малого и среднего бизнеса, в том числе производственного, к процессу сервисизации и цифровизации. Пэиллона и Дубруса (Peillona, Dubruca, 2019) детализируют препятствия для сервисизации производственных малых и средних предприятий, выделяя такие группы, как технические/технологические, организационные, связанные с человеческими ресурсами и клиентами. Однако указанные препятствия не являются непреодолимыми и могут рассматриваться, скорее, как стимул для развития, обеспечивающего конкурентоспособность на рынке. В частности, в работах (Humbeck, 
Pfähler, Wiedenmann, Herzwurm, 2019; Rapaccini, Mauro, Cinquini, Tenucci, 2019) pacсматриваются пути решения вопросов, связанных с переходом к бизнесу, основанному на услугах компаний, ориентированных на продукт.

Еще одну положительную сторону важности инновационности для МСП выявили Муштак, Гулл и Усман (Mushtaq, Gull, Usman, 2021). Исследователи установили, что результатом внедрения инноваций, включая современные ИКТ, является снижение асимметрии информации между МСП и банками. В итоге банки с большей вероятностью будут предоставлять кредиты и финансировать проекты и потребности в оборотном капитале инновационных МСП. То есть малые и средние предприятия с более широким доступом к новым технологиям и их использованием с большей вероятностью будут получать финансовые ресурсы от банков.

Несомненный интерес представляют результаты исследования (Ardito, Raby, Albino, Bertoldi, 2021), где изучены вопросы взаимосвязи цифровой и экологической ориентации компаний и их инновационности. Авторы показывают, что цифровая и экологическая ориентация оказывают прямое положительное влияние на эффективность инноваций в продуктах и процессах.

\section{МСП в Беларуси: планы и реальность}

Отечественные малые и средние предприятия играют весьма заметную роль в экономической жизни страны (Гудков, 2017). На

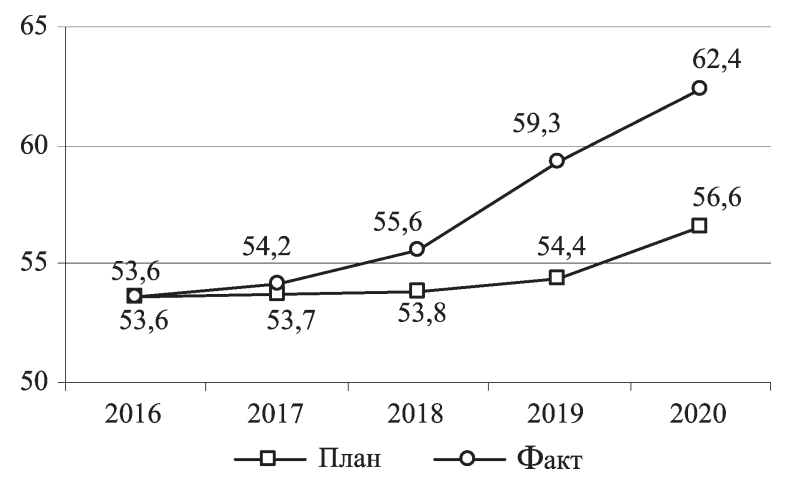

Рис. 1. Количество индивидуальных предпринимателей на 1 тыс. занятых в экономике, чел. их долю приходится более четверти ВВП и каждый третий занятый в белорусской экономике. В 2020 г. МСП обеспечили 81,7\% оптового товарооборота, $42,8 \%$ экспорта товаров и $45,6 \%$ импорта товаров ${ }^{1}$.

В то же время достигнутые параметры еще достаточно далеки не только от показателей развитых стран, но и от официально заявленного целевого уровня. В качестве базы для сравнения можно принять целевые показатели Государственной программы «Малое и среднее предпринимательство в Республике Беларусь на 2016-2020 годы», итоги выполнения которой подведены Министерством экономики Республики Беларусь.

По итогам реализации пятилетней госпрограммы из количественных параметров развития МСП обеспечен плановый уровень лишь по числу индивидуальных предпринимателей на 1 тыс. занятых в экономике (уровень выполнения составил $110,2 \%$ ) (рис. 1). В то же время количество юридических лиц субъектов малого и среднего предпринимательства на 1 тыс. занятых в экономике изменилось весьма несущественно (прирост составил $5,7 \%$ ), в результате чего отставание от планового уровня составило 17,3\% (рис. 2).

Результатом такого дисбаланса стал дефицит прогресса в изменении структуры за-

${ }^{1}$ Малое и среднее предпринимательство в Республике Беларусь. 2020. Минск: Национальный статистический комитет Республики Беларусь. URL: https://www.belstat.gov. by/upload/iblock/dd9/dd9792bfccf37c5c60d94f8fa1bb2507.pdf

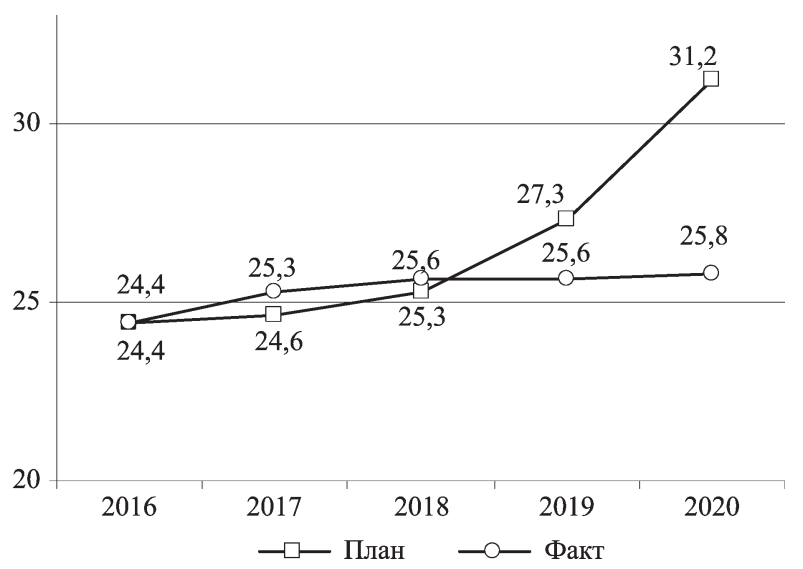

Рис. 2. Количество юридических лиц субъектов малого и среднего предпринимательства на 1 тыс. занятых в экономике, ед.

Источник. https://www.economy.gov.by/uploads/files/gos-progr-2016-2020/O-vypolnenii-gosudarstvennojprogrammy-Maloe-i-srednee-predprinimatelstvo-v-2016-2020.pdf 
нятости, предполагающий, в том числе переток рабочей силы из недостаточно эффективных предприятий и секторов экономики в малый бизнес. За 4 года отношение численности занятых в микроорганизациях, малых и средних организациях, а также индивидуальных предпринимателей и привлекаемых ими наемных лиц к общей численности занятых в экономике практически не изменилось (рис. 3), что может свидетельствовать о наличии определенного «разрыва» между декларируемыми целями и конкретными мерами по их реализации.

Наглядными маркерами наличия трудностей, связанных с процессом укрупнения бизнеса и его «взросления», являются показатели бизнес-демографии. В частности, отечественная статистика наглядно подтверждает тезис о том, что уровень «выживаемости» предприятий напрямую коррелирует с их размерами (рис. 4).

В качестве результирующих показателей анемичного развития сектора МСП можно рассматривать существенное отставание его доли от плановых значений в выручке от реализации, а также валовой добавленной стоимости (рис. 5). В частности, доля МСП в выручке от реализации за последние пять лет не только заметно меньше планового уровня (отставание составило 5,2 п. п.), но и наметилась тенденция к ее уменьшению - в 2018 г. доля дос-

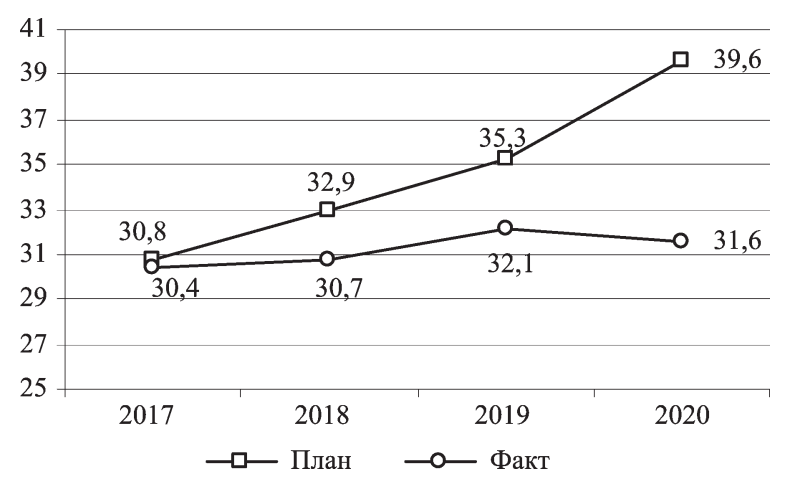

Рис. 3. Удельный вес занятых в микроорганизациях, малых и средних организациях, а также индивидуальных предпринимателей и привлекаемых ими наемных лиц в общей численности занятых в экономике, \%

Источник. https://www.economy.gov.by/uploads/ files/gos-progr-2016-2020/O-vypolnenii-gosudarstvennojprogrammy-Maloe-i-srednee-predprinimatelstvo-v-20162020.pdf

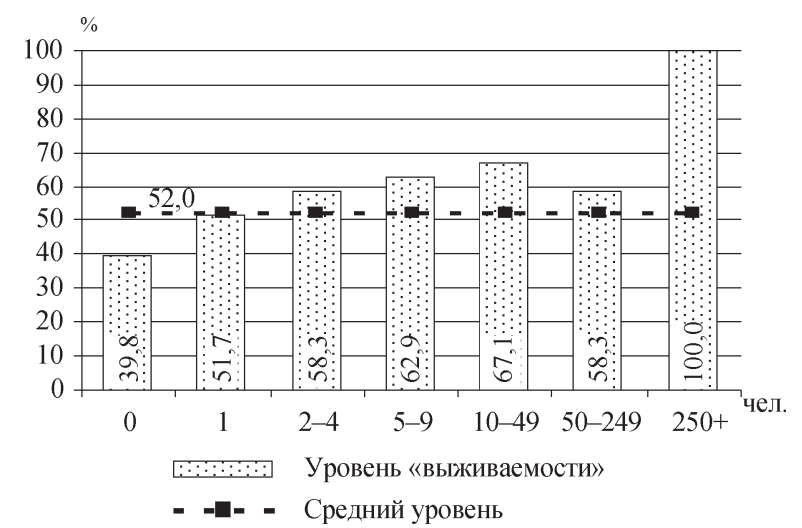

Примечание. По оси абсцисс приведен размер предприятия в 2015 г. (в зависимости от численности наемных работников).

Рис. 4. Уровень «выживаемости» «рожденных» в 2015 г. и «выживших» в 2020 г. предприятий по их размеру, \%

Источник. Бизнес-демография в Республике Беларусь: стат. бюллетень 2021. Минск: Национальный статистический комитет Республики Беларусь. С. 11.

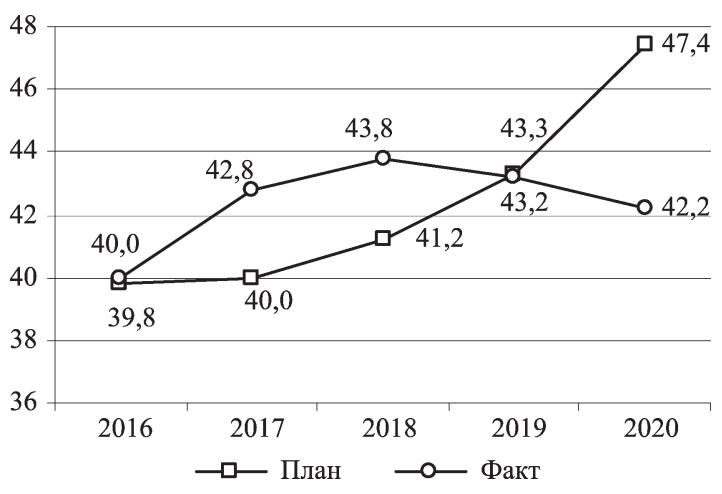

Выручка от реализации продукции, товаров, работ, услуг a)

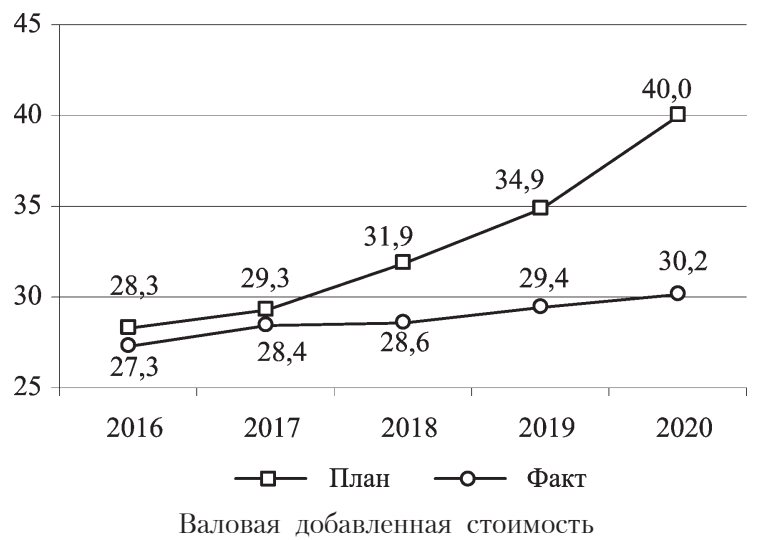

б)

Рис. 5. Удельный вес субъектов малого и среднего предпринимательства в экономике Республики Беларусь, \%

Источник. https://www.economy.gov.by/uploads/ files/gos-progr-2016-2020/O-vypolnenii-gosudarstvennojprogrammy-Maloe-i-srednee-predprinimatelstvo-v-20162020.pdf 
тигла $43,8 \%$, после чего снизилась до $42,2 \%$ в 2020 г. (рис. 5a).

Важно отметить, что инертность развития сектора МСП не является всеобщей тенденцией. В разрезе видов экономической деятельности ситуация существенно различается. В частности, серьезные метаморфозы претерпевает деятельность МСП, связанная с услугами в области информации и связи. Если в 2016 г. их удельный вес в совокупной выручке малого и среднего бизнеса составлял 2,3\%, то в 2020 г. уже $4,1 \%^{2}$. Рост в 1,7 раза, с одной стороны, отражает общие тренды в экономике Беларуси и мира, а с другой - демонстрирует наличие потенциала и возможных путей развития отечественных предпринимателей.

Подводя промежуточные итоги развития предпринимательства в Беларуси, следует акцентировать внимание на двух моментах.

Во-первых, можно констатировать перманентное торможение процесса «взросления» бизнеса, экономическая логика которого говорит о необходимости «эволюции» его субъектов, что подразумевает их переход на качественно более высокий уровень. Анализ имеющихся данных и тенденций позволяет сделать вывод: сектор МСП фактически застыл на достигнутом определенном уровне своего развития, что обуславливает необходимость комплексного пересмотра подходов к регулированию данной сферы со стороны государства.

Во-вторых, субъекты МСП, осуществляющие свою деятельность в быстро растущих секторах экономики (информационные технологии, высокоинтеллектуальные услуги), способны показывать весьма высокие результаты развития, существенно опережающие средние по сектору. Соответственно, одним из вариантов реализации потенциала малого и среднего предпринимательства может стать встраивание его в интеллектуальную экономику, формирование которой идет во всем мире с разной степенью интенсивности.

2 Рассчитано на основе: Малое и среднее предпринимательство в Республике Беларусь: стат. сборник. 2019. Минск: Национальный статистический комитет Республики Беларусь. C. 158; Малое и среднее предпринимательство в Республике Беларусь: стат. сборник. 2020. Минск: Национальный статистический комитет Республики Беларусь.

\section{Государственное видение путей развития МСП}

Сегодня экономические системы развитых стран мира находятся на этапе интеллектуализации, когда все более востребованным становится использование таких новых инструментов, как венчурный капитал и иные альтернативные источники финансирования, инновационные кластеры, участие в глобальном аутсорсинге услуг и т. д. При этом ключевым фактором производства становятся новые идеи, знания, обеспечивающие производство конкурентоспособной интеллектуальной продукции и, как следствие, закрепление предпринимательских структур страны в высокотехнологичных секторах мировой экономики.

Поскольку новое качество экономического роста непосредственно связано c активизацией процесса коммерциализации результатов научно-технической деятельности, несомненную актуальность приобретает задача повышения конкурентоспособности субъектов МСП на основе внедрения в бизнес-процессы инновационных технологий.

Развитие инновационных МСП происходит посредством уменьшения институциональных и прочих барьеров выхода на рынок в условиях динамичной конкуренции, которая позволяет предприятию относительно безболезненно переключаться на новые виды деятельности. Развитие технологий, в свою очередь, идет в контексте ускоренного накопления знаний, что является источником повышения предпринимательской активности. Прорывы в технологиях создают возможности для капитализации на базе эффективного использования комплекса новых знаний.

На всех уровнях государственного управления и в бизнес-сообществе закрепилось понимание инновационности как обязательного условия конкурентоспособности современного предприятия. При этом такой подход в контексте направлений, механизмов и параметров развития малого и среднего предпринимательства может применяться в несколько усеченном виде. В качестве иллюстрации данной ситуации целесообразно рассмотреть действующую вертикаль программных документов в Республике Беларусь. 
Основополагающим документом, очерчивающим долгосрочные перспективы развития страны, без сомнения, является Национальная стратегия устойчивого развития Республики Беларусь до 2035 года, в которой в контексте рассматриваемой проблематики можно выделить два основных посыла.

Во-первых, в стратегии одной из составляющих будущего облика Беларуси представлена развитая бизнес-среда с конкурентным предпринимательским сектором. Для достижения такого состояния намечена необходимость создания благоприятных условий для развития предпринимательства, включая совершенствование системы содействия развитию предпринимательства с учетом лучшей мировой практики, а также продвижение института саморегулирования ${ }^{3}$.

Во-вторых, особо подчеркивается важность поддержки инновационного предпринимательства и формирования широкого класса предпринимателей в высокотехнологичных отраслях экономики. Продекларирована необходимость государственного содействия изобретательской деятельности и инженерно-техническому творчеству, а также развитию технопарков, которые рассматриваются в качестве «инкубаторов» инновационного предпринимательства ${ }^{4}$.

В Программе социально-экономического развития Республики Беларусь на 20212025 годы обозначенные выше целевые установки также нашли свое отражение, хотя и в несколько видоизмененном виде ${ }^{5}$.

Что касается инновационного развития МСП, то оно в основном рассматривается в контексте модернизации отраслей либо же применительно к развитию соответствующей экосистемы. Вопросы становления малого инновационного предпринимательства затрагиваются достаточно фрагментарно и, по сути, сводятся к необходимости расширения практики предоставления инновационных ваучеров и грантов субъектам малого предпринимательства, а также планируемому внедрению венчурной экосистемы.

3 URL: http://www.economy.gov.by/uploads/files/ ObsugdaemNPA/NSUR-2035-1.pdf. C. 69.

${ }^{4}$ Там же. C. 40. P32100292
В части предметных вопросов развития малого и среднего предпринимательства на среднесрочную перспективу основной упор делается на улучшении деловой среды и механизмах совершенствования регуляторной практики. Одновременно в программе закреплено, что основным ее инструментом в части развития МСП станет реализация Государственной программы «Малое и среднее предпринимательство» на 2021-2025 годы ${ }^{6}$.

Изучение госпрограммы МСП позволяет говорить о ее некоторой дуалистичности в контексте инновационной повестки:

с одной стороны, в документе закреплены стремление к созданию инновационных организаций малого и среднего предпринимательства, ориентация на продвижение технологических инноваций, а также интеллектуализация этой сферы;

с другой стороны, в госпрограмме не предусмотрены действенные инструменты и стимулы, способные интенсифицировать достижение обозначенных выше целей.

В частности, целевые показатели госпрограммы МСП характеризуют лишь количественные параметры развития этой сферы экономики (например, доля МСП в валовой добавленной стоимости, выручке, экспорте товаров и услуг, инвестициях в общем объеме по стране или в регионах). Таким образом, качественная сторона развития субъектов МСП фактически остается вне фокуса государственного управления, что не позволяет говорить о выраженном векторе государственной политики в данной сфере.

Имеются узкие места и в системе государственной поддержки МСП. С одной стороны, в Беларуси создан ряд инструментов, направленных на развитие малого и среднего бизнеса ${ }^{7}$, включая систему финансовой поддержки инициатив в сфере молодежного предпринимательства (Климук, 2021), стимулирование инновационного предпринимательства (Дудинский, 2014) и др. В то же время результативность применения такого инструментария, вероятно, еще не достигла оп-

${ }^{6}$ URL: https://pravo.by/upload/docs/op/C22100056 1612386000.pdf

7 Манько М.А., Русак И.Н. 2020. Анализ роли поддержки малого и среднего предпринимательства в Республике Беларусь. Большая Евразия: Развитие, безопасность, сотрудничество: ежегодник. Вып. 3. С. 331-334. 
тимального уровня. В частности, результаты социологического исследования (Смирнова, 2020) показали, что лишь около половины белорусских предпринимателей знают о мерах государственной поддержки в данной сфере. Из всех опрошенных предпринимателей только 13,4\% получили финансовую поддержку, 5,1\% - имущественную, 8,4\% респондентов воспользовались формами стимулирования внешнеэкономической деятельности.

Это же исследование достаточно красноречиво иллюстрирует результативность реализуемой кластерной политики, которая в значительной степени и должна быть направлена на МСП. Так, лишь один из десяти (10,3\%) опрошенных представителей бизнеса знает о кластерах и кластерной политике государства, каждый четвертый $(25,9 \%)$ коечто о них слышал и ни один из респондентов не отметил, что его компания является участником такой формы кооперации.

\section{Инструментарий развития МСП в ЕС}

Исходя из того, что глобальное экономическое развитие неразрывно связано с процессами интеллектуализации и сервисизации, а также учитывая актуальность структурной трансформации экономики Беларуси, представляется целесообразным пересмотреть подходы к реализации государственной политики в отношении малого и среднего предпринимательства. В частности, государственная политика в отношении МСП должна стать более концентрированной и целеориентированной.

В этом контексте представляется весьма полезным обратиться к опыту экономически высокоразвитых стран, преуспевших в развитии малого и среднего бизнеса, изучение и адаптация которого к нашим условиям может помочь снизить сопутствующие издержки и улучшить ситуацию за счет использования апробированных передовых практик.

За основу предлагается взять опыт Европейского союза, что обусловлено несколькими причинами.

Во-первых, ЕС является наиболее продвинутым интеграционным объединением, опыт которого в значительной степени определил архитектуру Евразийского экономичес- кого союза. Учитывая, что значительная часть вопросов развития белорусских МСП прямо или опосредованно связана с функционированием общего рынка ЕАЭС, при выстраивании соответствующей государственной политики важно учитывать специфику работы в рамках интеграционного объединения.

Во-вторых, это экономически наиболее крупный и развитый альянс, с которым Беларусь граничит и осуществляет активную торговлю товарами и услугами. Соответственно, решения, принимаемые в ЕС, во многом определяют правила игры и конкурентоспособность как европейских предпринимателей, так и иных субъектов, имеющих связи с европейским рынком.

В Европейском союзе реализуется комплексный подход к вопросу экономического развития, увязанного в том числе с поддержкой и развитием МСП. Если говорить о текущем временном отрезке, то в качестве опорного элемента государственной экономической политики можно рассматривать «Новую промышленную стратегию для Европы», представленную Европейской комиссией в марте 2020 г. $^{8}$ и уточненную в 2021 г. с учетом воздействия на социальноэкономические процессы пандемии коронавирусной инфекции ${ }^{9}$. Названная стратегия посвящена определению общих целевых ориентиров развития экономики. В основе документа лежит концепция так называемого «двойного перехода», предусматривающего достижение климатической нейтральности и цифровое лидерство.

Предполагается, что названные составляющие двойной трансформации затронут все стороны жизни экономики и общества, включая создание новых продуктов, услуг и бизнес-моделей, новых типов рабочих мест, еще неизвестных сегодня, а также переход от линейного производства к экономике замкнутого цикла.

В самой промышленной стратегии закреплено, что она основана на предпринимательстве с широким многообразием его проявлений. Поэтому вполне закономерно, что одновременно с промышленной стра-

${ }^{8}$ European Commission. 2020. A New Industrial Strategy for Europe.

${ }_{9}^{9}$ European Commission. 2021. Updating the 2020 New Industrial Strategy: Building a stronger Single Market for Europe's recovery. 
тегией Еврокомиссия презентовала «Стратегию МСП для устойчивой и цифровой Европы», в которой весьма детально проработаны вопросы роли и форм участия европейского малого и среднего бизнеса в реализуемых преобразованиях. В частности, констатируется, что МСП «играют центральную роль в двойном переходе ЕС к устойчивой и цифровой экономике», а также то, что они должны его возглавить ${ }^{10}$.

В качестве цели стратегии ее разработчики видят такое состояние, при котором Европа должна быть наиболее привлекательным местом для начала малого бизне$\mathrm{ca}$, обеспечения его роста и расширения на едином рынке. В развитие указанного тезиса стратегия МСП закрепляет три основных направления приложения усилий:

1) создание потенциала и поддержка перехода к устойчивости и цифровизации;

2) снижение нормативной нагрузки и улучшение доступа к рынкам;

3) улучшение доступа к финансированию.

Каждое из указанных направлений достаточно масштабно и предполагает задействование разнообразных институций и механизмов. С позиции интереса к выстраиванию политики в отношении МСП, следует выделить ряд моментов.

Уже сегодня в ЕС около 25\% малых и средних предприятий предлагают экологически чистые товары и услуги, что хотя и является неплохим заделом, однако еще достаточно далеко от декларируемых целей грядущей трансформации. Одновременно имеются очевидные трудности с цифровизацией сектора. Так, только 17\% малых и средних предприятий успешно интегрировали цифровые технологии в свой бизнес, тогда как среди крупных европейских компаний этот показатель достиг 54\%, что свидетельствует о наличии определенных проблем в данном вопросе ${ }^{11}$.

Необходимость форсирования двойного перехода обусловила включение в стратегию МСП следующих мер:

${ }^{10}$ European Commission. 2020. An SME Strategy for a sustainable and digital Europe: https://ec.europa.eu/info/sites/ default/files/communication-sme-strategy-march-2020_en.pdf

11 European Commission. 2020. Unleashing the full potential of European SMEs.
- на базе Европейской сети поддержки предпринимательства (Enterprise Europe Network), объединяющей более 600 членов, будет организована работа консультантов по устойчивому развитию, ориентированных на оказание помощи малым и средним предприятиям в выборе направлений инвестирования, поиске коммерческих партнеров. Одновременно специализированные структуры, такие как Европейский центр знаний по ресурсоэффективности (European Resource Efficiency Knowledge Centre), окажут помощь в части внедрения энерго- и ресурсосберегающих технологий, доступа к ноу-хау и т. д.;

- 240 центров цифровых инноваций (Digital Innovation Hubs) будут поддерживать МСП в части интеграции цифровых инноваций в их продукты, бизнес-модели и процессы. Работа центров финансируется в рамках программы «Цифровая Европа» (Digital Europe Program), а также соответствующих фондов;

- Европейский инновационный совет (European Innovation Council) выделит 300 млн евро для поддержки прорывных инноваций достижения целей европейской «зеленой» сделки (Green Deal).

$\mathrm{C}$ целью сокращения бюрократических препятствий для ведения бизнеса запланировано существенное снижение барьеров на едином рынке. Сегодня 78\% европейских МСП воспринимают законодательство как сложное и обременительное, особенно изза различий в процедурах в государствахчленах. В этой связи Еврокомиссия запланировала к реализации пакет мер.

Так, для постоянного поиска способов упрощения законов ЕС и сокращения связанных избыточных затрат в интересах МСП будет создана «Платформа будущего» (Fit for Future Platform), представляющая собой группу экспертов высокого уровня, напрямую взаимодействующую с Еврокомиссией. В нормотворческой практике планируется внедрение принципа «один вошел - один вышел» (one-in, one-out), предполагающего, что введение любой регуляторной нормы будет сопровождаться отменой существующей аналогичной силы.

Будет проведена работа по активизации партнерства между приграничными регионами, задействована Директива об 
услугах, а также усовершенствована работа Единого цифрового шлюза (Single Digital Gateway) применительно к потребностям МСП. Отдельно выделено направление более широкого вовлечения МСП в работу космического и оборонного секторов, систему государственных закупок.

Немаловажное место в стратегии отводится решению проблемы задержки платежей в адрес МСП (только 40\% предприятий в ЕС получают оплату вовремя), а также обусловленных этим банкротств (это причина 1/4 банкротств МСП). Здесь основными инструментами Еврокомиссии станут Директива по борьбе с просрочкой платежей в коммерческих сделках (Late Payment Directive), а также Директива о превентивной реструктуризации и втором шансе (Directive on preventive restructuring frameworks and second chance).

В рамках облегчения доступа к финансовым ресурсам МСП планируется задействовать весьма широкий инструментарий. Так, для преодоления проблем использования венчурного финансирования предполагается усовершенствовать механизм технической экспертизы (Tech due diligence), необходимой для объективной оценки стартапов. Стимулирование проведения IPO для малого и среднего бизнеса будет осуществляться за счет инициативы ESCALAR. Еврокомиссия планирует запуск инициативы на основе блокчейна, которая должна помочь выпуску облигаций МСП и торговле ими по всей Европе с использованием европейской инфраструктуры блокчейн-сервисов. Намечено упрощение правил государственной помощи, оказываемой МСП, с задействованием национальных фондов, а также фондов InvestEU и Horizon.

Названные и ряд иных направлений государственного стимулирования развития МСП в ЕС складываются в целостный комплекс, объединяющий усилия национальных и наднациональных органов государственной власти, финансовых и нефинансовых институтов развития.

Важно отметить, что МСП также активно участвуют в популяризации собственной повестки. Например, в ЕС активно действует Европейский альянс цифровых MCП (European DIGITAL SME
Alliance), представляющий интересы 30 национальных и региональных ассоциаций МСП из стран - членов союза и соседних стран, включающий в свой состав более 45 тыс. субъектов.

Альянс представляет интересы своих членов в отношениях с институтами Европейского Союза, в том числе через лоббирование и участие в проектах, финансируемых ЕС, включен в процессы стандартизации, разрабатывает и продвигает документы стратегического характера ${ }^{12}$, а также занимается иными вопросами.

Деятельность подобных структур крайне важна с позиции вовлечения МСП в процесс выработки государственной политики и ее адаптации к изменениям в весьма подвижной бизнес-среде. В результате обеспечивается эффективная двусторонняя связь, помогающая соблюдать баланс интересов ее субъектов.

Исходя из изложенного, можно заключить, что сектор МСП в Беларуси пока полноценно не реализовал свой потенциал в качестве драйвера развития национальной экономики.

Прогресс в отдельных видах деятельности еще носит фрагментарный характер и существенно не влияет на общую динамику показателей сектора.

Программные документы, касающиеся социально-экономического развития Республики Беларусь, затрагивают перспективные вопросы развития МСП, однако в полной мере не подкреплены мероприятиями, требующими финансовых вложений.

До настоящего времени в стране нормативно не определен и, вследствие этого, статистически не наблюдаем сектор малого инновационного предпринимательства, играющий роль значимого поставщика инноваций в экономику развитых стран.

Сегодня отсутствует как таковая производственная кооперация частных фирм и производственных предприятий госсектора.

Совокупность названных факторов не позволяет в полной мере эффективно исполь-

${ }^{12}$ European DIGITAL SME Alliance. 2020. SKILLS FOR SMES STRATEGY 2030. 
зовать коммерческий потенциал МСП и делает невозможным их полноценное участие в процессе коммерциализации результатов научных исследований и разработок.

Важность инновационной составляющей в развитии МСП признается в подавляющем большинстве исследований, посвященных данной проблематике, как в Беларуси, так и за рубежом. Особенно этот вопрос актуализировался в контексте цифровизации и сервисизации значительной части бизнес-процессов.

В этом контексте важно учитывать и использовать в качестве образца европейские подходы к выстраиванию государственной политики в отношении МСП. При этом целесообразно задействовать потенциал Евразийского экономического союза, который должен стать объединяющей платформой для развития малого и среднего бизнеса в контексте сервисизации и интеллектуализации экономики.

Политику в отношении МСП следует выстраивать на сбалансированном задействовании потенциалов финансовых и нефинансовых институтов развития. Основным инструментом такой политики, бесспорно, должна стать система программирования, определяющая цели и задачи, закрепляющая конкретные мероприятия и ответственных за их выполнение, а также количественные и качественные параметры программируемых объектов в динамике.

\section{СПИСОК ЛИТЕРАТУРЫ (REFERENCES)}

Гудков С.В. 2017. Развитие малого и среднего предпринимательства в Беларуси в современных условиях. Проблемы экономики. № 1. C. 39-47. [Gudkov S.V. 2017. Development of small and medium-sized business in Belarus. Problemy ekonomiki. No 1. PP. 39-47. (In Russ.)]

Дудинский М.Э. 2014. Развитие инновационного предпринимательства Беларуси. Проблемы экономики. № 1. С. 63-73. [Dudinski M.E. 2014. Development of innovative business in Belarus. Problemy ekonomiki. No 1. PP. 63-73. (In Russ.)]

Климук В.В. 2021. Финансирование проектов инновационного молодежного предпринимательства в Республике Беларусь. Вестник Академии знаний. № 45(4). C. 335-345. [Klimuk V.V. 2021. Financing of innovative youth entrepreneurship projects in the Republic of Belarus. Vestnik Akademii znaniy. No 45(4). PP. 335-345. (In Russ.)]

Смирнова Р.А. 2020. Кластерные взаимодействия малого и среднего предпринимательства Беларуси: социологический анализ. Социологический альманах. № 11. С. 330-338. [Smirnova R.A. 2020. Cluster interactions of small and medium enterprises of Belarus: Sociological analysis. Sotsiologicheskiy al'manakh. No 11. PP. 330-338. (In Russ.)]

Томкович М.П. 2021. Сервисизация экономики Беларуси: альтернатива или предопределенность? Беларуская думка. № 8. С. 68-75. [Tomkovich M.P. 2021. Servitization of Belarus' economy: Alternative or predetermination? Belaruskaya dumka. No 8. PP. 68-75. (In Russ.)]

Шумпетер Й.А. 1982. Теория экономического развития. Москва: Прогресс. [Shumpeter J.A. 1982. Economic development theory. Moscow: Progress. (In Russ.)]

Ardito L., Raby S., Albino V., Bertoldi B. 2021. The duality of digital and environmental orientations in the context of SMEs: Implications for innovation performance. Journal of Business Research. Vol. 123. PP. 44-56.

Humbeck P., Pfähler K., Wiedenmann M., Herzwurm G. 2019. The Impact of Servitization and Digital Transformation - A Conceptual Extension of the IPOO-Framework. Procedia CIRP. Vol. 81. PP. 914-919.

Kim W.C., Mauborgne R. 2005. Blue ocean strategy. Harvard Business School Press.

Latifi M. A., Nikou S., Bouwman H. 2021. Business model innovation and firm performance: Exploring causal mechanisms in SMEs. Technovation. Vol. 107. 102274.

Mushtaq R., Gull A.A., Usman M. 2021. ICT adoption, innovation, and SMEs' access to finance. Telecommunications Policy. URL: https:// www.sciencedirect.com/science/article/abs/pii/ S0308596121001798

Peillona S., Dubruca N. 2019. Barriers to digital servitization in French manufacturing SMEs. Procedia CIRP. Vol. 83. PP. 146-150.

Rapaccini M., Mauro S. G., Cinquini L., Tenucci A. 2019. Servitization of SMEs through Strategic Alliances: a Case Study. Procedia CIRP. Vol. 83. PP. 176-181.

Rosenbusch N., Brinckmann J., Bausch A. 2011. Is innovation always beneficial? A meta-analysis of the relationship between innovation and performance in SMEs. Journal of Business Venturing. Vol. 26. Iss. 4. PP. 441-457.

Sağ S., Sezen B., Güzel M. 2016. Factors That Motivate or Prevent Adoption of Open Innovation by SMEs in Developing Countries and Policy Suggestions. Procedia - Social and Behavioral Sciences. Vol. 235. PP. 756-763. 
In citation: Belorusskiy Ekonomicheskiy zhurnal. 2021. No 4. PP. 138-148.

Belarusian Economic Journal. 2021. No 4. PP. 138-148.

\title{
DEVELOPMENT OF SMALL AND MEDIUM-SIZED BUSINESSES IN THE CONTEXT OF SERVICEIZATION AND INTELLECTUALIZATION OF THE BELARUSIAN ECONOMY
}

\author{
Yulia Medvedeva ${ }^{1}$, Mikhail Tomkavich ${ }^{2}$ \\ Author affiliation: ${ }^{1}$ Belarusian Institute of System Analysis and Information Support for Scientific \\ and Technical Sphere (Minsk, Belarus); \\ ${ }^{2}$ Research and Economic Institute of the Ministry of Economy of the Republic of Belarus \\ (Minsk, Belarus). \\ Corresponding author: Mikhail Tomkavich (tomkovich.michael@gmail.com).
}

ABSTRACT. Based on a review of literary sources, the article discusses the problems of the impact of innovation on small and medium-sized businesses (SMBs) in modern conditions, including the processes of serviceization and digitalization. An analysis of the development of SMBs in Belarus has been carried out. A number of state programmes have been studied with regard to the development of SMBs in this context. It has been established that they are rather fragmentary and focused mainly on the quantitative parameters of the development of this sector of the economy. The qualitative aspect of the development of SMBs actually remains outside the focus of state administration, which does not allow discussing a pronounced vector of state policy. Attention is paid to the functioning of the system of state support for SMBs. The article analyzes the toolkit for the development of SMBs in the European Union, including the implementation of the New Industrial Strategy and the concept of «double transition», which involves the achievement of climate neutrality and digital leadership.

KEYWORDS: Small and medium-sized businesses (SMBs), innovations, serviceization, intellectualization, foreign experience.

JEL-code: L26, O14, O30, O38.

DOI: $10.46782 / 1818-4510-2021-4-138-148$

Received 3.12.2021 